\title{
Improved survival in the largest national cohort of adults with cerebellar versus supratentorial low-grade astrocytomas
}

\author{
Jacob H. Bagley, B.S., ${ }^{1}$ Ranjith Babu, M.S., ${ }^{1}$ Allan H. Friedman, M.D., ${ }^{1}$ \\ and Cory Adamson, M.D., Ph.D., M.P.H., M.H.Sc. ${ }^{1-3}$ \\ ${ }^{1}$ Division of Neurosurgery, Department of Surgery; ${ }^{2}$ Department of Neurobiology, Duke University Medical \\ Center; and ${ }^{3}$ Durham Veterans Affairs Medical Center, Durham, North Carolina
}

\begin{abstract}
Object. Low-grade gliomas (LGGs) are indolent tumors that have the potential to dedifferentiate into malignant high-grade tumors. Recent studies have demonstrated that cerebellar low-grade tumors have a better prognosis than supratentorial tumors, although no study has focused on the risk factors for poor prognosis in cerebellar LGGs in adults. The authors of the current study aimed to address both of these concerns by using a large cohort derived from a national cancer registry and a smaller cohort derived from their institution's experience.

Methods. Adults with diagnosed Grade I and Grade II gliomas of the cerebellum were identified in the Surveillance, Epidemiology, and End Results (SEER) database. Multivariate Cox proportional hazard models were used to predict rates of survival, and the log-rank test was applied to evaluate differences in Kaplan-Meier survival curves. An institutional cohort was created by isolating all patients whose surgical pathology revealed an LGG of the cerebellum. Excluded from analysis were patients in whom a glioma was first diagnosed under the age of 18 years and those whose tumors could not be definitively determined to arise from the cerebellum.

Results. Data from the local cohort (11 patients) demonstrated that the most common presenting symptom was headache, which occurred in more than $70 \%$ of the cohort. Approximately half of the patients in this cohort had symptomatic improvement after treatment. Results from the SEER cohort (166 patients) revealed that adults with Grade I gliomas were slightly younger than those with Grade II tumors $(\mathrm{p}<0.01)$, but no other demographic differences were observed. Patients with Grade I tumors were twice as likely to undergo gross-total resection (54\% vs $21 \%$ ), and those with Grade II gliomas were much more likely to receive postoperative radiation (3\% vs $48 \%$ ). Five-year survival was greater in the patients with Grade I gliomas than in those with Grade II lesions (91\% vs 70\%). Multivariate analysis revealed that an age $\geq 40$ years (HR 7.30, 95\% CI 3.55-15.0, p < 0.0001) and Grade II tumors (HR 2.76, 95\% CI $1.12-6.84, p=0.028)$ were risk factors for death, whereas female sex was protective (HR $0.28,95 \%$ CI $0.14-0.59$, $\mathrm{p}<0.001)$. Log-rank tests revealed that a cerebellar location was protective $(\mathrm{p}<0.0001)$, but this relationship was only true for Grade II tumors $(\mathrm{p}<0.0001)$. Survival in patients with Grade I gliomas was not different based on the various lesion locations $(\mathrm{p}=0.21)$.

Conclusions. Taken together, adults with cerebellar WHO Grade I and II astrocytomas have a much more favorable survival curve than those with similar supratentorial tumors. Research demonstrates that the primary driver of this phenomenon is the improved survival in patients with cerebellar Grade II gliomas.
\end{abstract}

(http://thejns.org/doi/abs/10.3171/2012.12.FOCUS12343)

KEY WORDS
survival $\quad$ cerebellum $\quad$ low-grade glioma $\quad$ supratentorial astrocytoma

$\mathrm{D}$ ETERMINING the optimal treatment strategy for WHO Grade I and II gliomas, collectively referred to as LGGs, has been a goal of the neurosurgical research community since 1926 when Baily and Cushing ${ }^{1}$ first correlated prognosis with the histological appearance of the glioma. The following 80 years of clinical experience and research revealed that these tumors are relatively indolent, growing at approximately 4

\footnotetext{
Abbreviations used in this paper: GTR = gross-total resection; GIA = Grade I pilocytic astrocytoma; GIIA = Grade II fibrillary astrocytoma; $\mathrm{LGG}=$ low-grade glioma; NOS = not otherwise specified; SEER = Surveillance, Epidemiology, and End Results; STR = subtotal resection.
}

mm per year on average, ${ }^{16,17}$ and that patients with LGGs have a significantly higher survival rate than patients with higher-grade gliomas such as WHO Grade IV malignant glioblastoma multiforme. ${ }^{8,18,20}$ However, LGGs are a serious pathological entity because of where they can occur and their potential to dedifferentiate into malignant glioma. ${ }^{12,23}$ Although some authors have advocated a conservative watchful waiting approach to LGGs, ${ }^{3,4}$ there is growing consensus that GTR soon after presentation produces the greatest benefit for progression-free and overall survival. $7,11,13,14,19,22$

Not all LGGs are identical, however. Studies have focused on how prognosis may differ among LGGs with different growth rates, histological characteristics, presen- 


\section{J. H. Bagley et al.}

tations, and locations. ${ }^{6,16,17,21,24,26,27}$ The majority of extant research on these tumors in adults has focused on supratentorial examples, although some authors have suggested that LGGs of the cerebellum may have a different presentation and prognosis., ${ }^{9,11,24}$ Unfortunately, previous work examining cerebellar gliomas either has been focused on the pediatric population, in which these tumors are much more common, or has included very few cases. Thus, the ways in which cerebellar LGGs differ from supratentorial cases remain largely uncharacterized in adults.

In the current study we attempted to clarify the clinical characteristics of cerebellar LGGs by using 2 data sources: a national cohort of adults with cerebellar LGGs from the SEER database and a single-institution cohort of adults with cerebellar LGGs treated at Duke University. The national SEER database allowed us to determine whether established risk factors for prognosis in supratentorial LGGs, such as patient age, tumor grade, and extent of resection, behave similarly in cerebellar cases. Our institutional cohort allowed us to describe a variety of features of cerebellar LGGs that would be impossible to study using the available national database, which does not include information on patient presentations and responses to various therapies. Specifically, we were interested in describing ways in which patients with these tumors present and respond to treatment.

\section{Methods}

\section{National Cohort}

We used data from the SEER 18 Registries of the US National Cancer Institute SEER database (http://seer.can cer.gov). This comprehensive database, which is the gold standard for cancer registries, includes $28 \%$ of the population. ${ }^{15}$ Patients with pilocytic astrocytoma were selected using the ICD-O-3 code 9421; these patients constituted our Grade I cohort (GIA). Patients with astrocytoma NOS and fibrillary astrocytoma were selected using the ICDO-3 codes 9400 and 9420, respectively. Note that no cases of gemistocytic or protoplasmic astrocytomas in the cerebellum were found in the SEER database. We excluded from this group any patient who did not explicitly have a Grade II astrocytoma, since some higher-grade tumors were categorized as astrocytoma NOS. Patients in the remaining group composed our Grade II cohort (GIIA). The final cohorts were further defined by including only those patients who were 18 years of age and older and those with a primary tumor site in the cerebellum (site code C71.6). We excluded patients without pathological confirmation, those with multiple cancers, and those who were not active follow-up cases because their information was largely recorded at autopsy or on death certificates. We determined the location of the tumors with site codes C71.1, C71.2, C71.3, C71.4, and C71.6, which denoted frontal, temporal, parietal, occipital, and cerebellar locations, respectively. Using these codes, we dichotomized the Grade I and Grade II cohorts into cerebellar and supratentorial groups. The patients with supratentorial LGGs in the SEER database have been described in detail elsewhere. ${ }^{7}$ These patients -60 adults with supraten- torial Grade I gliomas and 1875 adults with supratentorial Grade II gliomas-are included in the current study only as an established comparison group to evaluate the association between cerebellar location and survival.

\section{Local Cohort}

A consecutive series of patients with cerebellar LGGs was created by searching our institution's pathology records for any patient with a histological diagnosis of pilocytic astrocytoma and fibrillary astrocytoma as an adult between 1994 and 2011. Patient demographic factors, such as sex, race, and age, were collected and recorded, as were the presenting symptomatology, surgical approach, and intervals between diagnosis, treatment, and last follow-up.

\section{Statistical Analysis}

Patient, tumor, and treatment characteristics were evaluated including age, sex, race, tumor size, and use of surgery and radiation therapy. Kaplan-Meier analysis was performed to assess overall survival, with the log-rank test used to evaluate differences between survival curves. Univariate and bivariate analyses were performed to determine the effect of various patient, tumor, and treatment variables on overall survival. Multivariate Cox proportional hazard models were used to calculate hazard ratios and $95 \%$ confidence intervals to investigate independent predictors of overall survival. Case-level data were concatenated and analyzed using SAS 9.3 (SAS Institute, Inc.), and a $\mathrm{p}$ value of 0.05 was used as the threshold of statistical significance.

\section{Results}

\section{National Cohort}

The SEER national cohort contained 71 patients with cerebellar GIAs and 95 patients with cerebellar GIIAs (Table 1). Patients with GIAs were typically younger than those with GIIAs $(\mathrm{p}<0.01)$. The most noticeable difference in age distribution occurred in the 18- to 20-year range $(29 \%$ of GIA vs $13 \%$ of GIIA, $\mathrm{p}<0.01)$. The 2 groups had a similar race distribution $(\mathrm{p}=0.16)$, with white $\mathrm{pa}-$ tients composing a majority in each group. There was no significant sex distribution difference between the GIA and GIIA groups $(p=0.15)$. While the data for tumor size was unavailable for a large proportion of patients (45\% of GIA and $79 \%$ of GIIA), available data revealed that the median tumor size, $30 \mathrm{~mm}$, was exactly the same in each group. The majority of both groups underwent resection (96\% and $87 \%$ of GIA and GIIA, respectively; $p=0.06$ ), with GTR being achieved at twice the rate in GIA as in GIIA (54\% vs $21 \%$ ), although information on the extent of resection was missing for $43 \%$ of patients with GIIA. In contrast to the GIA group, in which only $3 \%$ of patients received radiation, $48 \%$ of patients in the GIIA group received radiation as part of their initial treatment.

One year after diagnosis, $97 \%$ and $92 \%$ of patients in the GIA and GIIA groups, respectively, were still alive (Table 1). Five years after diagnosis, survival in the GIA group was $91 \%$, while in the GIIA group it was $69.5 \%$. Ten-year survival in the GIA and GIIA groups was $90 \%$ 
TABLE 1: Summary of patient, tumor, and treatment characteristics among adults with cerebellar WHO GIAs and GIIAs in the SEER database

\begin{tabular}{|c|c|c|}
\hline Variable & GIA & GIIA \\
\hline no. of patients & 71 & 95 \\
\hline median age in yrs (mean) & $25.0(30.9)$ & $34.0(38.0)$ \\
\hline \multicolumn{3}{|l|}{ age in yrs (no. of patients [\%]) } \\
\hline $18-20$ & $21(29)$ & $12(13)$ \\
\hline $21-30$ & $23(32)$ & $27(28)$ \\
\hline $31-40$ & $12(17)$ & $24(25)$ \\
\hline $41-50$ & $8(11)$ & $12(13)$ \\
\hline $51-60$ & $4(5)$ & $9(9)$ \\
\hline $61-70$ & $2(3)$ & $5(5)$ \\
\hline $71-80$ & $1(0.01)$ & $5(5)$ \\
\hline $81-90$ & $0(0)$ & $1(1)$ \\
\hline$>90$ & $0(0)$ & $0(0)$ \\
\hline \multicolumn{3}{|l|}{ race (no. of patients [\%]) } \\
\hline white & $60(86)$ & $84(88)$ \\
\hline black & $7(10)$ & $8(8)$ \\
\hline other & $4(5)$ & $3(3)$ \\
\hline \multicolumn{3}{|l|}{ sex (no. of patients [\%]) } \\
\hline M & $31(44)$ & $52(55)$ \\
\hline $\mathrm{F}$ & $40(56)$ & $43(45)$ \\
\hline median lesion size in $\mathrm{mm}$ & 30 & 30 \\
\hline $\begin{array}{l}\% \text { of patients for whom tumor size unknown } \\
\text { procedure (no. of patients [\%]) }\end{array}$ & 45 & 79 \\
\hline resection & $68(96)$ & $83(87)$ \\
\hline GTR & $38(54)$ & $20(21)$ \\
\hline STR & $23(32)$ & $22(23)$ \\
\hline extent unknown & $7(10)$ & $41(43)$ \\
\hline biopsy & $3(4)$ & $7(7)$ \\
\hline unknown & $0(0)$ & $5(5)$ \\
\hline \multicolumn{3}{|l|}{ postop treatment (no. of patients [\%]) } \\
\hline radiation & $2(3)$ & $46(48)$ \\
\hline no radiation & $69(97)$ & $46(48)$ \\
\hline unknown & $0(0)$ & $3(3)$ \\
\hline \multicolumn{3}{|l|}{ no. of deaths (\% surviving patients) } \\
\hline $1 \mathrm{yr}$ postdiagnosis & $2(97)$ & $7(92)$ \\
\hline 5 yrs postdiagnosis & $6(91)$ & $29(70)$ \\
\hline 10 yrs postdiagnosis & $7(90)$ & $34(64)$ \\
\hline
\end{tabular}

and $64 \%$, respectively. These differences in the survival rate between GIA and GIIA were significant at 5 and 10 years $(p<0.001)$. Several factors were significant predictors of survival when multivariate Cox proportional hazard models were used to control for the confounding effects of age, race, sex, radiation therapy, use of resection as treatment, and tumor grade (Table 2). Patients with an age $\geq 40$ years were 7.3 times more likely to die than patients younger than 40 years (95\% CI 3.55-15.00, p < 0.0001 ; Fig. 1). After 2 years, no one in the group younger than 40 years of age died, whereas the cohort 40 years old and older continued to experience a significant death rate.
TABLE 2: Multivariate analysis of patient and treatment factors in adults with cerebellar WHO GIAs and GIIAs from the SEER database

\begin{tabular}{lcc}
\hline \multicolumn{1}{c}{ Variable } & $\mathrm{HR}(95 \% \mathrm{Cl})$ & $\mathrm{p}$ Value \\
\hline $\begin{array}{l}\text { age in yrs } \\
<40\end{array}$ & 1 & - \\
$\geq 40$ & $7.30(3.55-15.0)$ & $<0.0001$ \\
sex & 1 & - \\
$\mathrm{M}$ & $0.28(0.14-0.59)$ & $<0.001$ \\
$\mathrm{~F}$ & 1 & - \\
race & & 0.076 \\
white & $2.75(0.90-8.42)$ & 0.068 \\
black & $3.18(0.92-11.0)$ & - \\
other & 1 & 0.21 \\
radiation therapy & $1.53(0.78-3.00)$ & - \\
no & & 0.88 \\
yes & 1 & - \\
procedure & & 0.028 \\
biopsy \\
resection \\
cerebellar tumor histology
\end{tabular}

Females had a $72 \%$ decreased risk of death compared with males (HR 0.28, 95\% CI 0.14-0.59, p < 0.001; Fig. 2). While there was no statistically significant difference in death rates among the racial groups examined, there was a trend toward decreased survival in the nonwhite groups ( $\mathrm{p}=0.08$ for black patients, $\mathrm{p}=0.07$ for patients of another race, both compared with white patients). Finally, the mortality risk in patients with GIIAs was more than double the risk in patients with GIAs (HR 2.76, 95\% CI $1.12-6.84, \mathrm{p}=0.028$ ).

Given that there was a clear difference in survival by tumor grade, we wanted to see how this difference was

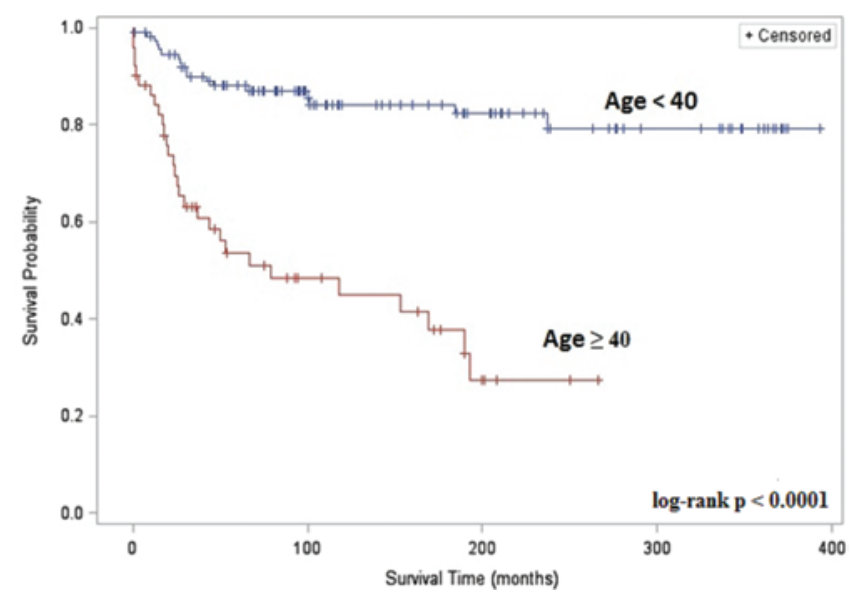

FIG. 1. Kaplan-Meier survival curves indicating worse survival in adults 40 years of age and older with cerebellar WHO GIAs and GIIAs from the SEER database (166 patients). 


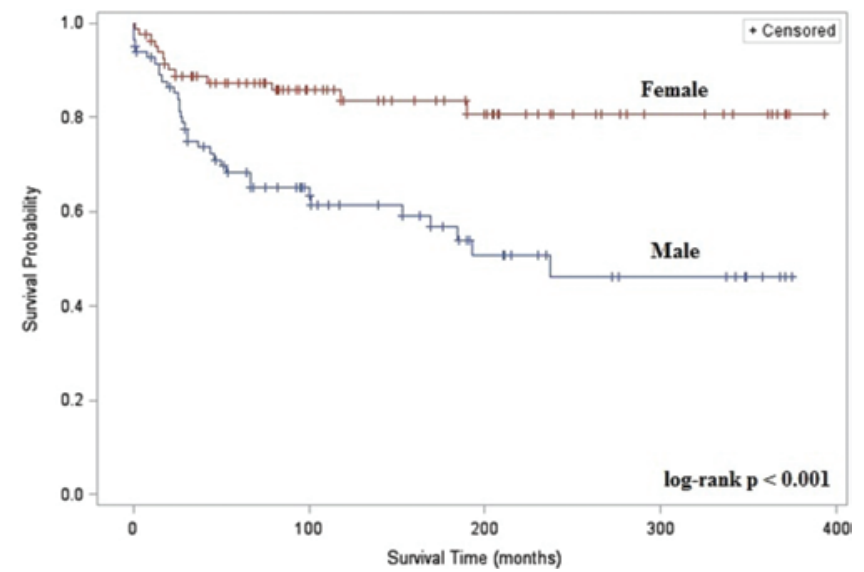

FIG. 2. Kaplan-Meier survival curves showing that adult females with Grade I or II cerebellar astrocytomas have improved survival.

affected by a supratentorial location (Fig. 3). The log-rank test revealed that the difference in survival was not significantly different for pilocytic astrocytomas that were cerebellar or supratentorial in location $(\mathrm{p}=0.21)$. This finding is in stark contrast to the decreased survival rate in patients with supratentorial GIIAs compared with that in patients with GIIAs in a cerebellar location $(\mathrm{p}<0.0001)$.

To evaluate the factors that may be driving this substantial difference in survival between supratentorial and cerebellar GIIAs, we compared these groups on a number of demographic, tumor, and treatment characteristics (Table 3). Patients with supratentorial Grade II gliomas tended to be older than those with cerebellar tumors (mean 41 vs 34 years, $\mathrm{p}<0.001$ ). Although a substantial number of patients in the SEER database lacked information about the size of their tumors, a comparison of available data revealed that supratentorial Grade II gliomas were significantly larger than gliomas occurring in the cerebellum (40 vs $30 \mathrm{~mm}, \mathrm{p}<0.01)$. In addition, a much

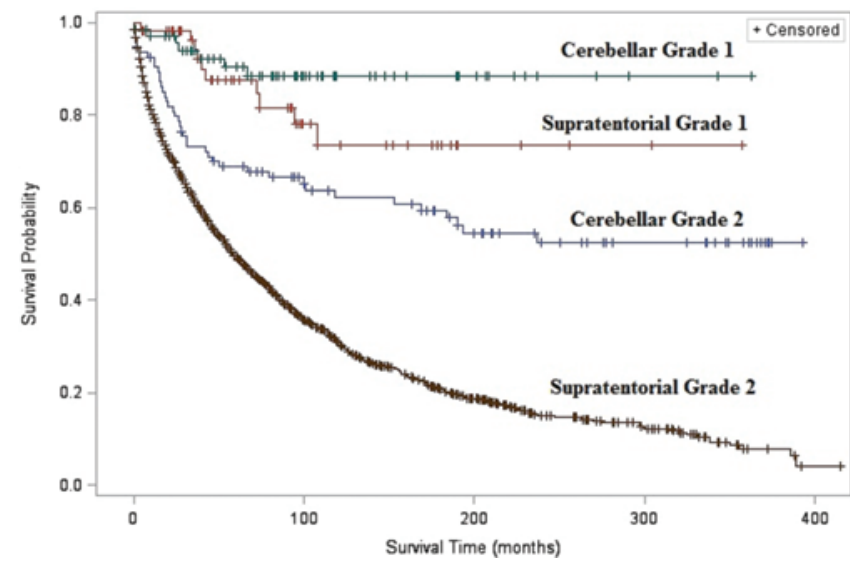

FIG. 3. Kaplan-Meier survival curves indicating that Grade II supratentorial astrocytomas are associated with worse survival than Grade II cerebellar astrocytomas, although a supratentorial location is not associated with worse survival for GIAs. Cerebellar Grade II tumors demonstrated worse survival than cerebellar Grade I tumors $(p<0.001)$. Survival in patients with cerebellar Grade I tumors was not significantly different from that in patients with supratentorial Grade I tumors $(p=0.21)$. Patients with supratentorial Grade II tumors had significantly worse survival than patients with cerebellar Grade II tumors $(p<0.0001)$.
TABLE 3: Patient, tumor, and treatment characteristics and survival in adults with supratentorial and cerebellar WHO GIIAs from the SEER database

\begin{tabular}{|c|c|c|}
\hline Variable & $\begin{array}{c}\text { Supratentorial } \\
\text { GIIA }\end{array}$ & $\begin{array}{c}\text { Cerebellar } \\
\text { GIIA }\end{array}$ \\
\hline no. of patients & 1875 & 95 \\
\hline median age in yrs (mean) & $41.0(45.0)$ & $34.0(38.0)$ \\
\hline \multicolumn{3}{|l|}{ age in years (no. of patients [\%]) } \\
\hline $18-20$ & $54(3)$ & $12(13)$ \\
\hline $21-30$ & $375(20)$ & $27(28)$ \\
\hline $31-40$ & $462(25)$ & $24(25)$ \\
\hline $41-50$ & $366(20)$ & $12(13)$ \\
\hline $51-60$ & $254(14)$ & $9(9)$ \\
\hline $61-70$ & $191(10)$ & $5(5)$ \\
\hline $71-80$ & $145(8)$ & $5(5)$ \\
\hline $81-90$ & $27(1)$ & $1(1)$ \\
\hline$>90$ & $1(0)$ & $0(0)$ \\
\hline \multicolumn{3}{|l|}{ race (no. of patients [\%]) } \\
\hline white & $1678(89)$ & $84(88)$ \\
\hline black & $109(6)$ & $8(8)$ \\
\hline other & $83(4)$ & $3(3)$ \\
\hline \multicolumn{3}{|l|}{ sex (no. of patients [\%]) } \\
\hline M & $1101(59)$ & $52(55)$ \\
\hline $\mathrm{F}$ & $774(41)$ & $43(45)$ \\
\hline median lesion size in $\mathrm{mm}$ & 40 & 30 \\
\hline $\begin{array}{l}\% \text { of patients for whom tumor size un- } \\
\text { known }\end{array}$ & 64 & 79 \\
\hline \multicolumn{3}{|l|}{ procedure (no. of patients [\%]) } \\
\hline resection & $1249(67)$ & $83(87)$ \\
\hline GTR & $348(19)$ & $20(21)$ \\
\hline STR & $432(23)$ & $22(23)$ \\
\hline extent unknown & $469(25)$ & $41(43)$ \\
\hline biopsy & $435(23)$ & $7(7)$ \\
\hline unknown & $191(10)$ & $5(5)$ \\
\hline \multicolumn{3}{|l|}{ postop treatment (no. of patients [\%]) } \\
\hline radiation & $1186(63)$ & $46(48)$ \\
\hline no radiation & $630(34)$ & $46(48)$ \\
\hline unknown & $59(3)$ & $3(3)$ \\
\hline \multicolumn{3}{|l|}{ no. of deaths (\% surviving patients) } \\
\hline $1 \mathrm{yr}$ postdiagnosis & $336(80)$ & $7(92)$ \\
\hline 5 yrs postdiagnosis & $879(53)$ & $29(70)$ \\
\hline 10 yrs postdiagnosis & $1125(40)$ & $34(64)$ \\
\hline
\end{tabular}

larger proportion of supratentorial Grade II gliomas were not treated with resection (33\% of supratentorial GIIA vs $13 \%$ of cerebellar GIIA, $\mathrm{p}<0.0001$ ), and many more patients with supratentorial GIIA tumors received postoperative radiation (63\% of those with supratentorial GIIAs vs $48 \%$ of those with cerebellar GIIAs, $\mathrm{p}<0.01$ ).

\section{Local Cohort}

Our institutional cohort of adults with cerebellar WHO Grade I and II low-grade astrocytomas consisted of 
25 patients. After excluding those not followed up by our institution (2 patients), those whose tumor origins could not be definitively classified as cerebellar (5 patients), and those whose tumors were first diagnosed when they were children (6 patients), the final cohort contained 12 adults, 11 of whom were treated for newly diagnosed tumors (Table 4). The median age at diagnosis was 28 years, with a range from 18 to 67 years. As in the national cohort, there was a predominance of white patients $(72 \%)$. In contrast to the national cohort, however, our patients included many more women than men ( $82 \%$ female). All cases in the local cohort involved GIAs. The predominant presenting symptom was headache, occurring in $73 \%$ of the sample, followed by dizziness/vertigo, which was present in $27 \%$. The interval between symptom onset and diagnosis ranged from 1 month to 20 years. The mean time from diagnosis to treatment was 1.5 months, and the most common treatment was GTR (8 cases), followed by STR ( 2 cases), and only 1 patient underwent biopsy. Despite more than 13 years of total follow-up for the entire cohort (follow-up range 1-34 months), there was not one incident of progression or recurrence. One of the patients in our cohort was seen at our institution for the treatment of a recurrent cerebellar low-grade astrocytoma.

\section{Discussion}

Data from this large national cohort reinforce prior assumptions that low-grade astrocytomas in the cerebellar region have a better prognosis than those located in supratentorial areas. Our findings clarify this understanding by defining the degree to which accepted risk factors, such as patient age and tumor grade, are influenced by a cerebellar lesion location. In addition, we describe the ways in which a cerebellar location for LGGs affects the clinical presentations and courses of adults with these tumors. We used 2 cohorts in our study: a local singleinstitution cohort of adults with cerebellar low-grade astrocytomas and a national cohort derived from the SEER database. In the national cohort, we found that although patients with Grade I and Grade II cerebellar astrocytomas resembled one another demographically, the patients with the Grade II gliomas were half as likely as those with Grade I astrocytomas to undergo GTR, probably because pilocytic astrocytomas are typically well circumscribed, easily resectable lesions, whereas Grade II astrocytomas can be more infiltrative (Table 1). As a consequence, almost half of the patients with Grade II astrocytomas had a course of postoperative radiation, since those who undergo STR are at higher risk for disease progression. ${ }^{13}$ Some studies have demonstrated that radiation therapy increases progression-free survival but not overall survival. ${ }^{13,25}$ However, a study of 179 supratentorial low-grade astrocytomas suggested that patients with certain risk factors, such as age $\geq 40$ years and treatment with STR, had a longer overall survival when they were treated with radiation. ${ }^{19}$ Thus, there may be subpopulations within the general low-grade astrocytoma population whose overall survival would benefit from radiation therapy. In our study, patients in the GIIA group who underwent STR and received radiation did have a non- significant trend toward increased survival $(p=0.43$, logrank test, data not shown). We were unable to ascertain the exact indications used to treat patients with radiation therapy in the national cohort.

As is the case for patients with other posterior fossa tumors, the local cohort with cerebellar low-grade astrocytomas typically presented with symptoms worrisome for obstructive hydrocephalus. While up to $80 \%$ of supratentorial cases will have associated seizures, the predominant symptom described by our patients was headache (Table 4). ${ }^{26}$ There was a large range for the prediagnosis chronicity of symptoms. Five of the 11 patients had symptoms lasting fewer than 5 months. Approximately half of the patients with cerebellar astrocytoma in our sample enjoyed some degree of symptomatic relief after their operations. Gross-total resection was possible for most of the patients, and STR or biopsy was largely determined by whether the tumor infiltrated the cerebellar peduncle and brainstem. Among the 11 cases, 2 tumors originated from the vermis, and those originating from the cerebellar hemispheres showed no obvious side preference. Three postoperative complications occurred including 1 wound infection, 1 pseudomeningocele, which did not require further surgical intervention, and 1 case of multiple small asymptomatic infarcts throughout the brain on postoperative MRI. After a mean follow-up interval of 15 months, there were no cases of progression and only 1 death, which occurred in a patient who had undergone STR, although the cause of death is unknown.

Data in the current study revealed a number of factors that increased the risk of death in adults with cerebellar low-grade astrocytomas. The most significant risk factor identified was an age $\geq 40$ years (Table 2; Fig. 1). Simply crossing the 40-year age threshold increased the risk of death by more than 7 times in adults with lowgrade astrocytoma, even after controlling for sex, race, tumor grade, resection, and use of radiation therapy. This risk factor has been identified in a number of studies focused on supratentorial gliomas. ${ }^{7,11}$ The difference in survival, compared with that in older patients, may be related to biologically more aggressive tumors, consistent with increased anaplasia on histological analysis according to some authors, ${ }^{2}$ and nontumoral factors, such as a higher comorbidity burden in older patients. A patient's sex was also determined to be a powerful risk-modifying factor. Adult females with cerebellar low-grade astrocytomas were $40 \%$ less likely to die than their male counterparts. This relationship has been described before in patients with supratentorial LGGs, although the reasons for this are still unclear.7 Interestingly, a systematic review of cerebellar astrocytomas of all grades from the pre-MRI era demonstrated no sex difference in survival, which suggests that early detection may play a role as well. ${ }^{10}$

Perhaps the most striking finding in our study is that a cerebellar location plays a clear role in the prognosis for Grade II astrocytomas in adults. Other studies have demonstrated that patients with cerebellar LGGs as a whole have more favorable survival rates than similar patients with supratentorial tumors. ${ }^{11}$ When we stratified patients by tumor grade and then examined the effect of lesion location, we found that the driving force behind the previous- 
J. H. Bagley et al.

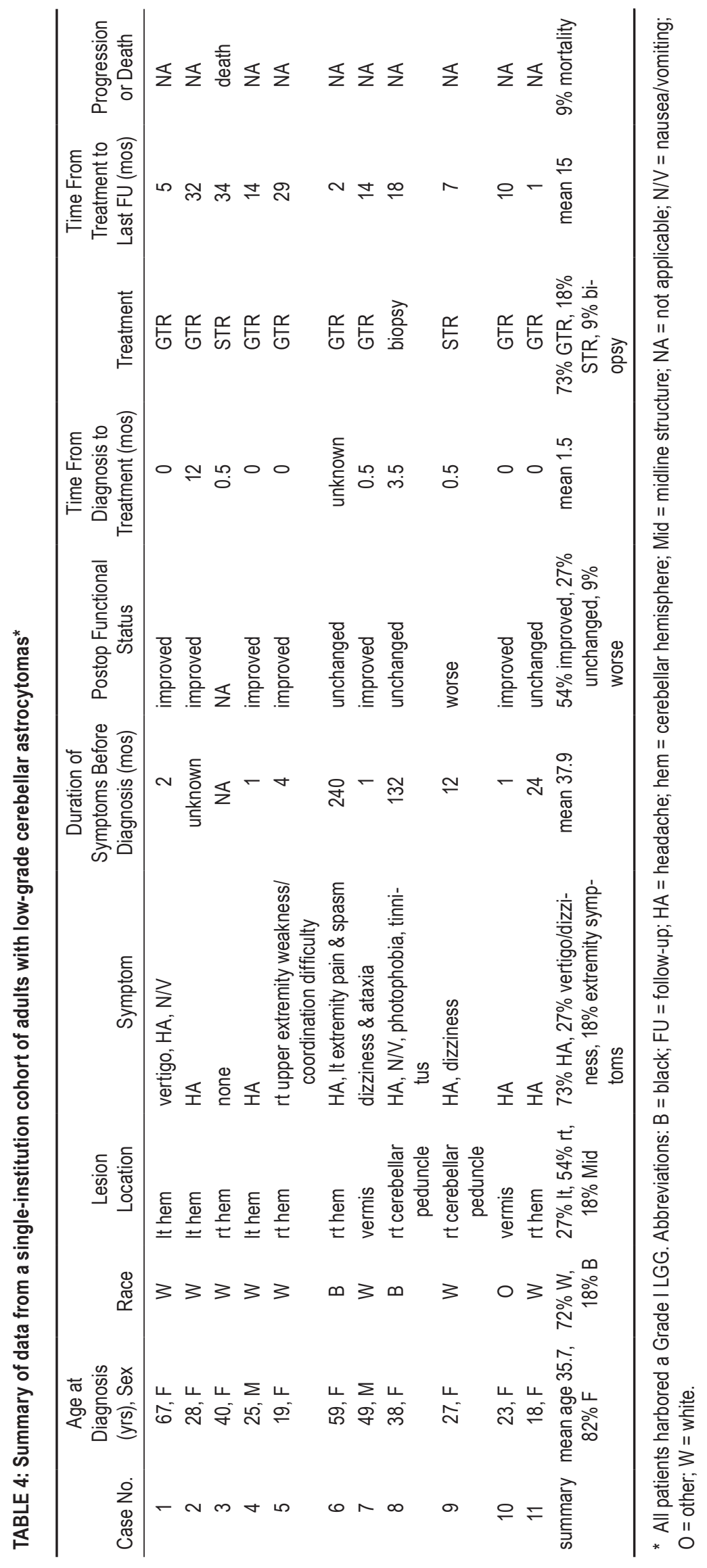


ly observed improved survival of patients with cerebellar tumors was the enormous difference in survival between those with supratentorial and those with cerebellar Grade II astrocytomas. At 10 years after diagnosis, only about $40 \%$ of patients with supratentorial Grade II tumors had survived, whereas more than $60 \%$ of patients with cerebellar Grade II tumors were still alive. Moreover, the death rate in the supratentorial Grade II group was largely constant, with a linear portion of the Kaplan-Meier survival curve in the period after 10 years of follow-up. In contrast, after 10 years of follow-up, the cerebellar Grade II group had strikingly few deaths. It is impossible to say at this point whether this finding is attributable to differences in recurrence or malignant transformation.

To better address the potential causes for the large difference in survival patterns between patients with supratentorial and those with cerebellar Grade II gliomas, we examined a number potential demographic, tumorassociated, and treatment factors (Table 3). While the 2 groups had significantly different age distributions, we do not believe that this difference could be a large driver of the distinct observed survival rates, since the bulk of the age disparity was attributable to a much larger young adult population in the cerebellar GIIA cohort. The 2 factors that appeared to have the greatest potential influence on survival were tumor size and the use of resection for treatment. Both supratentorial and cerebellar Grade II astrocytoma groups had a similar rate of GTR and STR. However, many fewer patients with supratentorial Grade II gliomas underwent resection than those with cerebellar Grade II gliomas (67\% of supratentorial vs $87 \%$ of cerebellar, $\mathrm{p}<0.0001)$. The reason for this difference is unclear but probably attributable to the fact that the Grade II gliomas in the supratentorial region were $10 \mathrm{~mm}$ larger at presentation than those in the cerebellum. Their increased size and consequently the increased chance for involvement of eloquent cortex probably dissuades many surgeons from pursuing resection in these patients. While the use of awake brain mapping to preserve functional cortex has been demonstrated to improve extent of resection and survival, many who treat patients with these tumors do not regularly use this technique. The reluctance to use resection in the treatment of these patients is probably a large driver in the survival disparity between supratentorial and cerebellar Grade II gliomas.

At least 2 other possibilities exist for the observed difference in survival between adults with cerebellar and those with supratentorial astrocytomas. First, incidentally found astrocytomas-defined by Potts et al. ${ }^{21}$ and Pallud et al. ${ }^{17}$ as "a finding on imaging that was obtained for a reason not attributable to the glioma, such as trauma or headache"-tend to have a better prognosis. Potts and colleagues $^{21}$ recently reported that patients with incidental supratentorial LGGs have a significantly higher rate of survival. Since the diagnosis in the majority of patients in the local cohort was rendered during a workup for headache, it is likely that the proportion of incidental tumors (using the above definition) is much greater among cerebellar gliomas than among supratentorial ones, in which seizures are a common presenting symptom. Second, it is possible that Grade II astrocytomas in the cerebellum have a lower rate of malignant dedifferentiation than supratentorial examples, although there is no evidence to support this possibility at the moment. Further studies are clearly warranted to address these various theoretical causes of this phenomenon.

This study represents the largest analysis of the risk factors determining survival in adults with cerebellar lowgrade astrocytomas. In the adult population, these tumors are rare entities, so previous research has either focused on the pediatric population or included very few patients. By using the SEER database, we were able to create a large national cohort of patients and decrease the potential bias that occurs when reporting results from a single institution. However, there are several reasons why the data presented here require cautious interpretation. Various clinical and tumor variables could not be gathered from the SEER database, including patient performance status, duration of symptoms, imaging characteristics, and progression. Moreover, treatment characteristics, such as the dose of radiotherapy, chemotherapy, or surgical approach, could not be determined. A substantial number of patients in the national cohort had unknown tumor size $(45 \%$ and $79 \%$ of those with GIAs and GIIAs, respectively). Slightly more than $40 \%$ of GIIAs in this cohort had an unknown extent of resection. This amount of missing data could confound survival analyses; therefore, we decided to not explicitly compare the effects of GTR and STR on survival. Finally, as there is no central pathology review of cases in the SEER database, misdiagnosed cases may be present. However, studies examining other cancers have demonstrated an acceptable rate of concordance between a patient's pathology and its coded representation in a cancer registry. ${ }^{5}$ Despite these limitations, these data add considerably to our understanding of cerebellar low-grade astrocytoma in adults.

\section{Conclusions}

Together, cerebellar WHO Grade I and II astrocytomas in adults have a much more favorable survival curve than similar supratentorial tumors. Our research demonstrates that the primary driver of this phenomenon is the improved survival in patients with cerebellar Grade II gliomas relative to that in patients with supratentorial Grade II gliomas. Our findings suggest that the worse survival of patients with supratentorial Grade II gliomas may be attributable to the decreased use of resection in such cases.

\section{Disclosure}

The authors report no conflict of interest concerning the materials or methods used in this study or the findings specified in this paper.

Author contributions to the study and manuscript preparation include the following. Conception and design: all authors. Acquisition of data: Bagley, Babu. Analysis and interpretation of data: Adamson, Bagley, Babu. Drafting the article: Adamson, Bagley, Babu. Critically revising the article: all authors. Reviewed submitted version of manuscript: all authors. Approved the final version of the manuscript on behalf of all authors: Adamson. Statistical analysis: Adamson, Bagley, Babu. Study supervision: Friedman. 


\section{References}

1. Bailey P, Cushing H: A Classification of the Tumors of the Glioma Group on a Histogenic Basis With a Correlated Study of Prognosis. Philadelphia: JB Lippincott, 1926

2. Barker FG II, Chang SM, Huhn SL, Davis RL, Gutin PH, McDermott MW, et al: Age and the risk of anaplasia in magnetic resonance-nonenhancing supratentorial cerebral tumors. Cancer 80:936-941, 1997

3. Brasil Caseiras G, Ciccarelli O, Altmann DR, Benton CE, Tozer DJ, Tofts PS, et al: Low-grade gliomas: six-month tumor growth predicts patient outcome better than admission tumor volume, relative cerebral blood volume, and apparent diffusion coefficient. Radiology 253:505-512, 2009

4. Cairncross JG, Laperriere NJ: Low-grade glioma. To treat or not to treat? Arch Neurol 46:1238-1239, 1989

5. Castillo MS, Davis FG, Surawicz T, Bruner JM, Bigner S, Coons $\mathrm{S}$, et al: Consistency of primary brain tumor diagnoses and codes in cancer surveillance systems. Neuroepidemiology 23:85-93, 2004

6. Chang EF, Smith JS, Chang SM, Lamborn KR, Prados MD, Butowski N, et al: Preoperative prognostic classification system for hemispheric low-grade gliomas in adults. Clinical article. J Neurosurg 109:817-824, 2008

7. Claus EB, Black PM: Survival rates and patterns of care for patients diagnosed with supratentorial low-grade gliomas: data from the SEER program, 1973-2001. Cancer 106:13581363, 2006

8. Davis FG, Freels S, Grutsch J, Barlas S, Brem S: Survival rates in patients with primary malignant brain tumors stratified by patient age and tumor histological type: an analysis based on Surveillance, Epidemiology, and End Results (SEER) data, 1973-1991. J Neurosurg 88:1-10, 1998

9. Hassounah M, Siqueira EB, Haider A, Gray A: Cerebellar astrocytoma: report of 13 cases aged over 20 years and review of the literature. Br J Neurosurg 10:365-371, 1996

10. Ilgren EB, Stiller CA: Cerebellar astrocytomas. Clinical characteristics and prognostic indices. J Neurooncol 4:293-308, 1987

11. Johnson DR, Brown PD, Galanis E, Hammack JE: Pilocytic astrocytoma survival in adults: analysis of the Surveillance, Epidemiology, and End Results program of the National Cancer Institute. J Neurooncol 108:187-193, 2012

12. Jung TY, Jung S, Moon JH, Kim IY, Moon KS, Jang WY: Early prognostic factors related to progression and malignant transformation of low-grade gliomas. Clin Neurol Neurosurg 113:752-757, 2011

13. Karim AB, Afra D, Cornu P, Bleehan N, Schraub S, De Witte $\mathrm{O}$, et al: Randomized trial on the efficacy of radiotherapy for cerebral low-grade glioma in the adult: European Organization for Research and Treatment of Cancer Study 22845 with the Medical Research Council study BRO4: an interim analysis. Int J Radiat Oncol Biol Phys 52:316-324, 2002

14. Keles GE, Lamborn KR, Berger MS: Low-grade hemispheric gliomas in adults: a critical review of extent of resection as a factor influencing outcome. J Neurosurg 95:735-745, 2001

15. Malin JL, Kahn KL, Adams J, Kwan L, Laouri M, Ganz PA: Validity of cancer registry data for measuring the quality of breast cancer care. J Natl Cancer Inst 94:835-844, 2002
16. Mandonnet E, Delattre JY, Tanguy ML, Swanson KR, Carpentier AF, Duffau H, et al: Continuous growth of mean tumor diameter in a subset of grade II gliomas. Ann Neurol 53: 524-528, 2003

17. Pallud J, Mandonnet E, Duffau H, Kujas M, Guillevin R, Galanaud D, et al: Prognostic value of initial magnetic resonance imaging growth rates for World Health Organization grade II gliomas. Ann Neurol 60:380-383, 2006

18. Peraud A, Ansari H, Bise K, Reulen HJ: Clinical outcome of supratentorial astrocytoma WHO grade II. Acta Neurochir (Wien) 140:1213-1222, 1998

19. Philippon JH, Clemenceau SH, Fauchon FH, Foncin JF: Supratentorial low-grade astrocytomas in adults. Neurosurgery 32:554-559, 1993

20. Piepmeier J, Christopher S, Spencer D, Byrne T, Kim J, Knisel JP, et al: Variations in the natural history and survival of patients with supratentorial low-grade astrocytomas. Neurosurgery 38:872-879, 1996

21. Potts MB, Smith JS, Molinaro AM, Berger MS: Natural history and surgical management of incidentally discovered lowgrade gliomas. Clinical article. J Neurosurg 116:365-372, 2012

22. Smith JS, Chang EF, Lamborn KR, Chang SM, Prados MD, Cha $S$, et al: Role of extent of resection in the long-term outcome of low-grade hemispheric gliomas. J Clin Oncol 26: 1338-1345, 2008

23. Soffietti R, Chiò A, Giordana MT, Vasario E, Schiffer D: Prognostic factors in well-differentiated cerebral astrocytomas in the adult. Neurosurgery 24:686-692, 1989

24. Stokland T, Liu JF, Ironside JW, Ellison DW, Taylor R, Robinson KJ, et al: A multivariate analysis of factors determining tumor progression in childhood low-grade glioma: a population-based cohort study (CCLG CNS9702). Neuro Oncol 12:1257-1268, 2010

25. van den Bent MJ, Afra D, de Witte O, Ben Hassel M, Schraub S, Hoang-Xuan K, et al: Long-term efficacy of early versus delayed radiotherapy for low-grade astrocytoma and oligodendroglioma in adults: the EORTC 22845 randomised trial. Lancet 366:985-990, 2005

26. van Veelen ML, Avezaat CJ, Kros JM, van Putten W, Vecht C: Supratentorial low grade astrocytoma: prognostic factors, dedifferentiation, and the issue of early versus late surgery. J Neurol Neurosurg Psychiatry 64:581-587, 1998

27. Youland RS, Brown PD, Giannini C, Parney IF, Uhm JH, Laack NN: Adult low-grade glioma: 19-year experience at a single institution. Am J Clin Oncol [epub ahead of print], 2012

Manuscript submitted October 15, 2012.

Accepted December 11, 2012.

Please include this information when citing this paper: DOI: 10.3171/2012.12.FOCUS12343.

Address correspondence to: Cory Adamson, M.D., Ph.D., M.P.H., M.H.Sc., Division of Neurosurgery, Duke University Medical Center, Box 2624, Durham, North Carolina 27710. email: cory. adamson@duke.edu. 